\title{
Dislocations as a Tool for Nanostructuring Advanced Materials
}

\author{
Vladyslav Turlo
}

check for

updates

Citation: Turlo, V. Dislocations as a Tool for Nanostructuring Advanced Materials. Physchem 2021, 1, 225-231. https://doi.org/10.3390/ physchem 1030016

Academic Editors: Jacinto Sá and Tullio Scopigno

Received: 26 June 2021

Accepted: 10 September 2021

Published: 26 September 2021

Publisher's Note: MDPI stays neutral with regard to jurisdictional claims in published maps and institutional affiliations.

Copyright: (c) 2021 by the author Licensee MDPI, Basel, Switzerland. This article is an open access article distributed under the terms and conditions of the Creative Commons Attribution (CC BY) license (https:/ / creativecommons.org/licenses/by/ $4.0 /)$.
Laboratory for Advanced Materials Processing (LAMP), Swiss Federal Laboratories for Materials Science and Technology (Empa), CH-3602 Thun, Switzerland; vladyslav.turlo@empa.ch

\begin{abstract}
Dislocations present unique opportunities for nanostructuring advanced structural and functional materials due to the recent discoveries of linear complexions thermodynamically stable nanoscale features with unique chemistry and structure confined at dislocations. The formation of such features is driven by solute segregation near the dislocation core and results in the stabilization of dislocations, altering mechanical, thermodynamic, and transport properties of the final material. This perspective article gives an overview of the recent discoveries and predictions made by highresolution experimental characterization techniques, as well as large-scale atomistic simulations in the newly emerging field of linear complexions.
\end{abstract}

Keywords: dislocations; segregation; phase transitions; linear complexions; atomistic simulations

\section{Introduction}

Dislocations are linear defects controlling many mechanical and transport properties of metal-based materials. One meter cubed of heavily deformed metal can contain up to one light-year of total dislocation length [1], offering limitless opportunities for nanoscale material design. As the world is moving towards multi-component materials such as superalloys and high-entropy alloys for their unique properties and performance, an understanding of the interaction of dislocations with alloying elements and impurities becomes crucial for controlling and tailoring such properties and performance. While theoretical predictions for the structure of a dislocation core and its decoration by solutes and impurities were developed decades ago, the experimental validation of such predictions was not possible due to the nanoscale size of the dislocation core requiring 3D atomic-scale characterization inside of the bulk material. High-resolution transmission electron microscopy (HRTEM) allows for atomic-scale characterization of crystal defects and, in the case of grain boundary decoration by solutes, HRTEM imaging enabled the discovery of many kinds of thermodynamically stable chemical and structural states called grain boundary complexions [2]. In the case of dislocation decoration by solutes, however, HRTEM imaging would require much more effort, with extra tilting and twisting required to find the right angle for characterizing the dislocation core area with atomic precision. Only in recent years, the nanoscale specimen preparation techniques in combination with advanced 3D atom-scale characterization techniques, such as atom probe tomography (APT), in addition to HRTEM enabled a comprehensive analysis of the dislocation-solute interactions, leading to many discoveries beyond the predictions of classical theories $[1,3,4]$.

\section{Dislocation Solute Decoration and Discovery of Linear Complexions}

Let's take for example a simple Pt-Au system with just two face-centered cubic (fcc) solid phases present on the phase diagram; one is Pt-rich and another is Au-rich, and both are solid solutions. No bulk structural transitions are expected in such a system, yet a slight difference in lattice constants of $\mathrm{Pt}$ and $\mathrm{Au}$ ensures solute segregation to the dislocation core, making Pt-Au a perfect system for isolating and investigating the "chemical life" of crystal defects such as dislocations. By investigating a Pt-7 at.\% Au sample magnetron-sputtered and annealed at $1300 \mathrm{~K}$, Zhou et al. [2] have recently demonstrated a high complexity 
associated with the solute decoration of such defects. The peak solute concentrations at four distinct types of defects, which included the dislocation array in a low-angle grain boundary, a glissile dislocation, a stacking fault tetrahedron, and a Frank loop, were measured by APT to be 29, 33, 42, and 63 at.\% Au, respectively, well above the bulk composition of $7 \mathrm{at} . \% \mathrm{Au}$. Such variation of solute concentration at the nanoscale creates many opportunities for local microstructure designs while going beyond the classical theories of Cottrell segregation, to dislocations and Suzuki segregation to stacking faults. Thus, more theoretical work is required to connect the nanoscale structure of crystalline defects with the corresponding solute decoration so that the proper predictions can be made for similar binary metallic alloys, superalloys, and high-entropy alloys. However, what about more complex systems where structural transitions are possible?

Fe-Mn alloys serve as an example of a system with more possibilities for structural transitions, as these materials can maintain a body-centered cubic (bcc) structure called ferrite or an fcc structure called austenite depending on the temperature and composition. Kuzmina et al. [3] used both APT and TEM to investigate the Fe-9 at.\% Mn ferrite single-crystal plastically deformed and annealed at three different temperatures. These authors discovered thermodynamically stable nanoscale precipitate arrays along the dislocation lines, which they called linear complexions. The compositions of such precipitates corresponded to the austenite phase from the equilibrium phase diagram for all three temperatures investigated, and the assumption of an austenite phase was also supported by a weak reflection of an fcc structure in a diffraction analysis conducted with TEM. However, the mechanism of formation of such thermodynamically stable precipitates with the composition well above the one predicted for the Cottrell segregation to edge dislocation was not clear until a detailed thermodynamic analysis of the Fe-Mn system was performed by Kwiatkowski de Silva et al. [4] in 2018. According to this analysis, the dislocation segregation zone with elevated composition undergoes spinodal decomposition in the bcc lattice, leading to the formation of alternating zones of low and high Mn composition. The zones with high Mn content were acting as a precursor to the structural bcc-fcc transformation and formation of nanoscale precipitate arrays observed experimentally. By analyzing APT data, Kwiatkowski de Silva et al. also confirmed that such spinodal decomposition occurs for other defects such as low-angle and high-angle grain boundaries, making these spinodal states another kind of complexion with the possibility of partial structural transition to the austenite phase. However, spinodal decomposition is not necessary to call these states complexions as long as they are confined to the defect and are in thermodynamic equilibrium with the matrix phase.

\section{The Predictive Power of Atomistic Simulations}

Inspired by so many discoveries made by analyzing just the two systems of Pt-Au and Fe-Mn, one would want to perform more systematic studies also involving binary systems with more complex phase diagrams. Unfortunately, such studies would require tremendous experimental effort in exploring the local chemical ordering near the dislocation core associated with the solute segregation and formation of intermetallic phases. In contrast, atomistic simulations allow for systematic studies when leveraged with high-performance/cloud computing and can provide the missing information about solute-defect interactions, all while acting as a digital 3D microscope capturing structural and chemical transitions at the nanoscale. Atomistic simulations were successfully applied to predict the local structure, chemistry, and properties of grain boundary complexions. This lead to the design of advanced structural and functional materials in particular thermally-stable nanocrystalline metals, with superior properties and performance in various environments [1]. The hybrid Monte Carlo/molecular dynamics method is essential for capturing complexion states and their transitions in atomistic simulations. Molecular dynamics allows for structural relaxation while Monte Carlo allows for chemical relaxation, together allowing for complete thermodynamic equilibration of the system at a given thermodynamic ensemble. The variance-constrained semi-grand canonical ensemble is necessary for the thermodynamic 
equilibration of the system at a given temperature, pressure, and composition, capable of capturing phase/complexion coexistence at the nanoscale [5].

Turlo and Rupert [6] were the first to capture linear complexion states with atomistic simulations by analyzing solute segregation and nanoscale phase transformations at edge dislocations in the bcc Fe-Ni system. By exploring the wide space of temperatures and compositions, these authors discovered that $\mathrm{Ni}$ segregation to the compression side of the dislocation core can lead to the formation of nanoscale precipitates composed of one (B2-FeNi), two (B2-FeNi and $\mathrm{L}_{0}-\mathrm{FeNi}$ ), or even three (B2-FeNi, $\mathrm{L}_{0}-\mathrm{FeNi}$ and $\mathrm{L1}_{2}-\mathrm{FeNi}_{3}$ ) intermetallic phases, with the $\mathrm{B} 2$ phase being metastable and not present on the bulk Fe-Ni phase diagram [6,7]. By using the high-performance Monte Carlo code developed by Sadigh et al. [5], these authors were able to extend the size of the systems along the dislocation lines, proving the presence of a narrow region on the equilibrium phase diagram corresponding to the formation of linear complexions [6]. As shown in Figure 1, such linear complexion states were formed at the dislocation segregation zones (compression side) as nanoscale regions of elevated composition (Figure 1a) with local chemical ordering corresponding to B2 (red) and $\mathrm{L} 1_{0}$ intermetallic phases (green) in Figure $1 \mathrm{~b}, \mathrm{c}$.
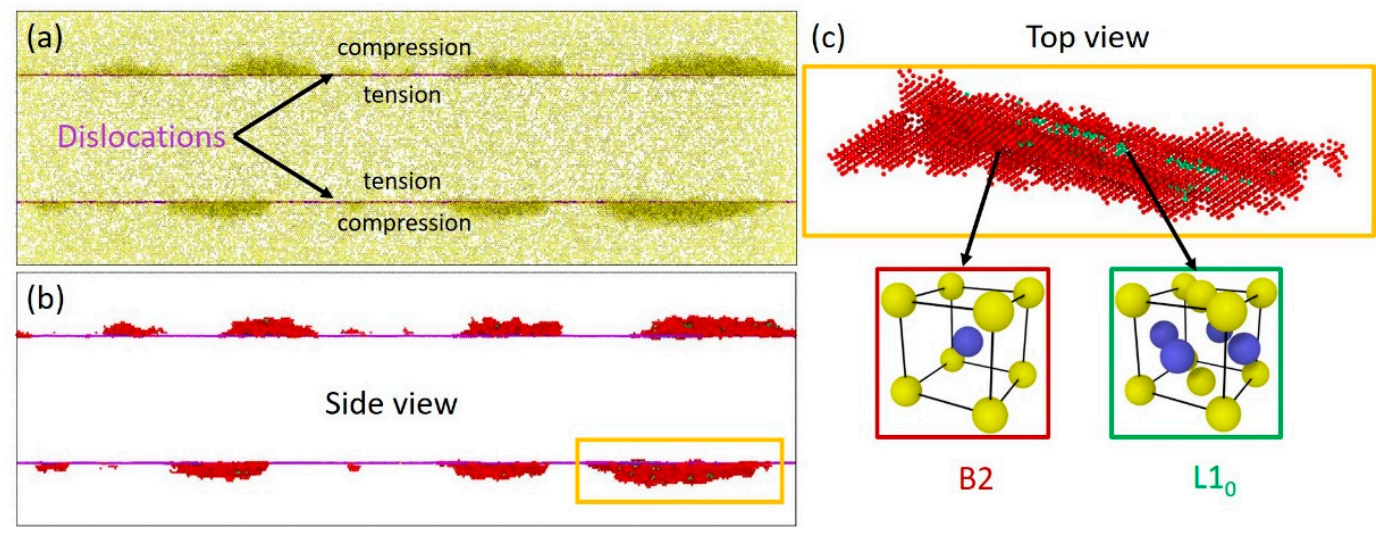

Figure 1. The side view of the Fe-2 at.\% Ni sample annealed at $500 \mathrm{~K}$ with (a) only solute atoms shown (yellow) and (b) only ordered phases shown (red-B2, green-L1 $)$. (c) Top view of the precipitate highlighted by the yellow box in (b). Magenta lines indicate the positions of dislocations in $(\mathbf{a}, \mathbf{b})$. The dislocation line is not shown in (c) as it would cover the $\mathrm{L} 1_{0}$ phase.

While the coexistence of these intermetallic phases can be explained just by analyzing the bulk and interface energies [7], the role of the dislocation stress field in such nanoscale phase transformations was not clear. Shear deformation of a sample such as the one shown in Figure 1 promotes the dislocation slip away from the linear complexions, leading to inverse lattice distortive transformation from the $\mathrm{L}_{0}$ back to the $\mathrm{B} 2$ phase [8]. Due to the periodic boundary conditions, the dislocations were able to come back to their original location, recovering the $\mathrm{L}_{0}$ phase inside of the $\mathrm{B} 2$ precipitates, as shown in Figure $1 \mathrm{c}$. These results show that the presence of the dislocation stress field is essential for the nanoscale two-phase coexistence and stability of linear complexions. Due to the metastable nature of B2 phase precipitates, they will be dissolved in the matrix as soon as dislocation moves away from the linear complexions. Overall, the formation of linear complexions is the result of the complex interplay between bulk, interface, and strain energies impacted by solute-dislocation interactions at the nanoscale. Beyond simple line defects that can act as a source for segregation and structural transition, dislocations can also have a more two-dimensional shape. The most common example of this is found in fcc alloys, where the dislocation core is usually comprised of two Shockley partial dislocations with a stacking fault in-between them.

By exploring a wide range of compositions and temperatures in $\mathrm{Ni}-\mathrm{Fe}, \mathrm{Al}-\mathrm{Zr}, \mathrm{Al}-\mathrm{Cu}$, and $\mathrm{Cu}-\mathrm{Zr}$ (the first element is a matrix, the second element is a dopant), Turlo and Rupert were able to identify three different classes of linear complexions based on their effect on the dislocation core [9]. The first type, namely nanoparticle array linear complexions, was observed 
in the Ni-Fe and Al-Zr systems with nanoscale precipitate arrays of stable and metastable $\mathrm{L}_{2}$ phases formed along the partial dislocations. This type of complexion also includes previously discussed linear complexions in the Fe-Ni and Fe-Mn systems and makes no impact on the structure of the dislocation core. The second type of linear complexions, namely platelet array linear complexions, has been observed in the Al-Cu alloys as arrays of Guinier-Preston (GP) zones with thickness from one to three atomic planes forming along the partial dislocations. Such nanoscale platelets are commonly observed in quenched and annealed supersaturated $\mathrm{Al}$ alloys as the precursors to the formation of stable intermetallic phases and are commonly used for precipitation hardening of $\mathrm{Al}$ alloys. While GP zones are usually metastable, in this case, these features can be stabilized by the dislocations, which could also act as the nucleation sides for future, mature GP zones due to the elevated composition of the dislocation segregation zone. In turn, GP zones push partial dislocations out of their slip planes in the direction of their growth, thus restructuring the dislocation core. The third type of linear complexions, namely stacking fault linear complexions, has been found in the $\mathrm{Cu}-\mathrm{Zr}$ system in the form of a nanoribbon of the $\mathrm{Cu}_{5} \mathrm{Zr}$ intermetallic phase that replaces the original stacking fault. The formation of such complexions is the result of Suzuki-type solute segregation to the stacking fault followed by structural and chemical reordering, resulting in delocalization of the dislocation core. This is the most extreme case of complexion transformation at a dislocation, in which stacking faults and partial dislocations cannot be recognized in their original form after the complexion formation. As an extension of previous results to multicomponent alloys, it is possible to design coexisting linear complexions by doping $\mathrm{Al}$ with both $\mathrm{Cu}$ and $\mathrm{Zr}$ atoms. In this way, the $\mathrm{L}_{2}-\mathrm{Al}_{3} \mathrm{Zr}$ nanoscale precipitates form in addition to GP zones, with both growing through the dislocation slip plane [9]. Such coexisting linear complexions demonstrate the complex nature of solute-dislocation interactions in multicomponent alloys while highlighting the new avenues for nanostructuring advanced materials.

\section{Dislocation Stabilization and Its Potential Applications}

Dislocations are commonly thought of as metastable and highly mobile crystal defects that are expected to easily glide and eventually annihilate/disappear at planar defects such as grain boundaries and free surfaces when the system is approaching thermodynamic equilibrium. However, the recent results of Turlo and Rupert highlight the extreme impact of linear complexion states on stabilization and pinning of edge dislocations in the bcc Fe-Ni alloys, in turn affecting the mechanical properties and performance of such advanced nanostructured materials [8]. Similar simulation results were also reported by Pascuet et al. [10] while analyzing solute segregation to screw dislocations in the bcc Fe doped with $\mathrm{Ni}, \mathrm{Mn}, \mathrm{Cu}, \mathrm{Si}$, and $\mathrm{P}$, highlighting the strong pinning of screw dislocations decorated by solutes. The stable complexion-like states predicted by Pascuet et al. [10] were similar to those reported by Kuzmina et al. [3] and received experimental validation by means of the APT analysis of reactor pressure vessel steels under ion and neutron irradiation [11]. The stabilization of dislocation networks by solute segregation and linear complexion formation in bcc Fe explains the strain aging behavior during mechanical testing of the Fe-Mn samples reported by Kwiatkowski de Silva et al. [12].

At the same time, solute segregation to stacking faults in fcc metals and formation of nanoscale ribbons of the second phase could significantly affect high-temperature creep resistance, as it has been recently demonstrated for several commercial Ni-based superalloys $[13,14]$. For example, $\mathrm{Co}$ and $\mathrm{Cr}$ segregation and $\mathrm{Ni}$ and $\mathrm{Al}$ depletion at extrinsic and intrinsic stacking faults in the ME3 superalloy leads to the formation of nanoribbons of gamma phase inside gamma prime precipitates, promoting the growth of nanotwins and reducing the creep resistance of the alloy [13]. In contrast, the segregation of $\mathrm{Ti}, \mathrm{Ta}$, and $\mathrm{Nb}$ to the extrinsic stacking faults in the ME501 superalloy led to the formation of eta phase nanoribbons [13], while the segregation of $C o$ and $W$ to the intrinsic stacking faults in the LSHR superalloy led to the formation of kappa phase nanoribbons [14], both inhibiting the growth of nanotwins and improving the creep resistance of these superalloys. As all these 
nanoribbons are formed under load during high-temperature deformation, they are not in thermodynamic equilibrium with the matrix phase; thus, they are proposed to be classified by Smith et al. as a new class of dynamic complexions [13].

Such dynamical complexions could also appear in other highly non-equilibrium conditions associated with, for example, laser and electron beam additive manufacturing, which is characterized by rapid heating and cooling cycles during the fabrication process, strongly promoting defect formation in the resulting microstructure [15]. This is especially true for metals and alloys with high thermal conductivity and low laser absorptivity, in which high residual stresses lead to the formation of large networks of geometrically necessary dislocations $[16,17]$. While the fabrication process could potentially promote the formation of dynamic complexions, the post-process heat treatment could promote the formation of more stable nanoscale precipitates of intermetallic phases [18], which, if confined to dislocations, would form a basis for linear complexions. Due to the complex interplay between kinetics and thermodynamics of complexion (phase) transformations at the nanoscale, the fabrication of samples with stable dislocation networks remains challenging and would require tremendous research efforts in the following decade on both experimental and theoretical sides. In particular, the integration and transfer of information about linear complexions in the multiscale mechanics modeling workflows are needed, effectively combining atomistic simulations with discrete dislocation dynamics and crystal plasticity models to establish a clear connection between the nanoscale structure and chemistry of linear complexions, and the mechanical performance of the resulting material [19].

At the same time, dislocation stabilization opens up many opportunities for tailoring other properties besides mechanical, such as thermodynamic, and transport properties as well as the ability to design new types of nanostructured materials for various applications. Stable dislocation networks would provide fast diffusion paths and impurity/solute trapping areas [20-22], which could lead, for example, to the design of new materials for hydrogen transportation and storage [23], a critical roadblock towards the green economy. Stable dislocations may also be considered as a new tool for designing novel Si-based devices (light emitters, manipulators of biomolecules, thermoelectric generators, etc.) due to their ability to maintain and carry charge along the dislocation lines while doped with metals and impurities [24]. Moreover, dislocation lines in p-type silicon are demonstrated supermetallic conductivity, acting as tiny nanowires with eight orders of magnitude faster electron transport than in the surrounding Si matrix [25]. However, the applications of dislocation stabilizations and linear complexion formation in micro-and nanoelectronics would not be limited to Si. For example, Ishida et al. [26] demonstrated that dislocation movement in gold nanocontacts leads to an electrical conductance oscillation, which could have a detrimental impact, for example, on ultra-low power electric devices and nanowire photovoltaic devices. In this case, alloying of nanocontacts and stabilization of dislocations by solute decoration could solve the problem. At the same time, transformation to linear complexions in the form of nanoscale precipitates after cold rolling and annealing could substantially reduce the presence of alloying elements in the matrix material, which could strongly improve the electrical conductivity of the overall material, as was demonstrated for an Al-Mg-Si conductor alloy [27].

\section{Key Challenges and Concluding Remarks}

It is likely that the next decade of research in such a new exciting field of research as linear complexions will lead to many discoveries and the design of new materials for structural and functional applications. However, several challenges should be overcome in the nearest future:

- The experimental characterization techniques must be able to identify chemicallyordered phases in three dimensions at the nanoscale to validate the modeling results. For example, the aberration-corrected atomic-resolution imaging combined with the 
state-of-the-art energy-dispersive X-ray (EDX) spectroscopy could be used for such a goal [13].

- The modeling techniques must be improved to capture not only thermodynamics, but also the kinetics of defect segregation and complexion transitions at the nanoscale, which would lead to many discoveries associated with dynamic complexions discussed above. Diffusive molecular dynamics [28] is one of the most promising methods right now for modeling the diffusion-driven processes at the nanoscale. While resolved over large time scales, diffusive molecular dynamics can be also used as an alternative method to the hybrid molecular dynamics/Monte Carlo method to characterize solute segregation to crystal defects [3]. Unfortunately, the quantitative agreement of both methods with experiments strongly depends on the ability of interatomic potential to represent the real system, which brings us to the next point.

- The accuracy of atomistic simulations should be improved, in particular, by upgrading the high-performance Monte Carlo and diffusive molecular dynamics codes [5,28] to enable a larger variety of classical interatomic potentials as well as recently emerging machine learning/neural network interatomic potentials approaching ab initio accuracy [29]. In addition, both methods should enable the efficient equilibration of complex multicomponent systems such as superalloys, medium- and high-entropy alloys, etc.

While not complete, this list represents the essential first steps towards enabling a comprehensive fundamental investigation of linear complexions and their impact on various properties of advanced materials. The author believes that, in the long history of metallurgy lasting thousands of years, linear complexions were always present in the tools and metal parts, waiting until now to be discovered. Indeed, the cold working followed by annealing that was used by Kuzmina et al. [1] to prepare samples with linear complexions has a long history starting from the development of copper and bronze metallurgy in the Near East many thousands of years ago [30]. In addition, even after the development of alloying and casting processes in an open mold during 5000 to 3000 BC, the final shape of the metal parts and tools was acquired by cold hammering and annealing, significantly improving their mechanical performance. Unfortunately, non-destructive state-of-the-art nanoscale characterization techniques are not yet capable of analyzing ancient samples to confirm the presence of stable dislocations decorated by solutes, which would be another challenge for the new decade to overcome.

Funding: This research received no external funding.

Acknowledgments: We thank Timothy Rupert (University of California, Irvine) for fruitful discussions.

Conflicts of Interest: The authors declare no conflict of interest.

\section{References}

1. Kuzmina, M.; Herbig, M.; Ponge, D.; Sandlöbes, S.; Raabe, D. Linear complexions: Confined chemical and structural states at dislocations. Science 2015, 349, 1080-1083. [CrossRef]

2. Cantwell, P.R.; Frolov, T.; Rupert, T.J.; Krause, A.R.; Marvel, C.J.; Rohrer, G.S.; Rickman, J.M.; Harmer, M.P. Grain Boundary Complexion Transitions. Annu. Rev. Mater. Res. 2020, 50, 465-492. [CrossRef]

3. Zhou, X.; Mianroodi, J.R.; Kwiatkowski da Silva, A.; Koenig, T.; Thompson, G.B.; Shanthraj, P.; Ponge, D.; Gault, B.; Svendsen, B.; Raabe, D. The hidden structure dependence of the chemical life of dislocations. Sci. Adv. 2021, 7, eabf0563. [CrossRef] [PubMed]

4. Kwiatkowski Da Silva, A.; Ponge, D.; Peng, Z.; Inden, G.; Lu, Y.; Breen, A.; Gault, B.; Raabe, D. Phase nucleation through confined spinodal fluctuations at crystal defects evidenced in Fe-Mn alloys. Nat. Commun. 2018, 9, 1137. [CrossRef] [PubMed]

5. Sadigh, B.; Erhart, P.; Stukowski, A.; Caro, A.; Martinez, E.; Zepeda-Ruiz, L. Scalable parallel Monte Carlo algorithm for atomistic simulations of precipitation in alloys. Phys. Rev. B 2012, 85, 184203. [CrossRef]

6. Turlo, V.; Rupert, T.J. Dislocation-assisted linear complexion formation driven by segregation. Scr. Mater. 2018, 154, 25-29. [CrossRef]

7. Turlo, V.; Rupert, T.J. Linear Complexions: Metastable Phase Formation and Coexistence at Dislocations. Phys. Rev. Lett. 2019, 122, 126102. [CrossRef]

8. Turlo, V.; Rupert, T.J. Interdependent Linear Complexion Structure and Dislocation Mechanics in Fe-Ni. Crystals 2020, 10, 1128. [CrossRef] 
9. Turlo, V.; Rupert, T.J. Prediction of a wide variety of linear complexions in face centered cubic alloys. Acta Mater. 2020, 185, 129-141. [CrossRef]

10. Pascuet, M.I.; Monnet, G.; Bonny, G.; Martínez, E.; Lim, J.J.H.; Burke, M.G.; Malerba, L. Solute precipitation on a screw dislocation and its effects on dislocation mobility in bcc Fe. J. Nucl. Mater. 2019, 519, 265-273. [CrossRef]

11. Odette, G.R.; Almirall, N.; Wells, P.B.; Yamamoto, T. Precipitation in reactor pressure vessel steels under ion and neutron irradiation: On the role of segregated network dislocations. Acta Mater. 2021, 212, 116922. [CrossRef]

12. Kwiatkowski da Silva, A.; Leyson, G.; Kuzmina, M.; Ponge, D.; Herbig, M.; Sandlöbes, S.; Gault, B.; Neugebauer, J.; Raabe, D. Confined chemical and structural states at dislocations in Fe-9wt\%Mn steels: A correlative TEM-atom probe study combined with multiscale modelling. Acta Mater. 2017, 124, 305-315. [CrossRef]

13. Smith, T.M.; Esser, B.D.; Antolin, N.; Carlsson, A.; Williams, R.E.A.; Wessman, A.; Hanlon, T.; Fraser, H.L.; Windl, W.; McComb, D.W.; et al. Phase transformation strengthening of high-temperature superalloys. Nat. Commun. 2016, 7, 13434. [CrossRef]

14. Smith, T.M.; Good, B.S.; Gabb, T.P.; Esser, B.D.; Egan, A.J.; Evans, L.J.; McComb, D.W.; Mills, M.J. Effect of stacking fault segregation and local phase transformations on creep strength in Ni-base superalloys. Acta Mater. 2019, 172, 55-65. [CrossRef]

15. Hooper, P.A. Melt pool temperature and cooling rates in laser powder bed fusion. Addit. Manuf. 2018, 22, 548-559. [CrossRef]

16. Wang, G.; Ouyang, H.; Fan, C.; Guo, Q.; Li, Z.; Yan, W.; Li, Z. The origin of high-density dislocations in additively manufactured metals. Mater. Res. Lett. 2020, 8, 283-290. [CrossRef]

17. Yamanaka, K.; Kuroda, A.; Ito, M.; Mori, M.; Bian, H.; Shobu, T.; Sato, S.; Chiba, A. Quantifying the dislocation structures of additively manufactured Ti-6Al-4V alloys using X-ray diffraction line profile analysis. Addit. Manuf. 2021, 37, 101678. [CrossRef]

18. Tan, C.; Zhou, K.; Ma, W.; Zhang, P.; Liu, M.; Kuang, T. Microstructural evolution, nanoprecipitation behavior and mechanical properties of selective laser melted high-performance grade 300 maraging steel. Mater. Des. 2017, 134, 23-34. [CrossRef]

19. Bertin, N.; Sills, R.B.; Cai, W. Frontiers in the Simulation of Dislocations. Annu. Rev. Mater. Res. 2020, 50, 437-464. [CrossRef]

20. Love, G.R. Dislocation pipe diffusion. Acta Metall. 1964, 12, 731-737. [CrossRef]

21. Lin, J.; Luo, X.; Zhong, X.; Zhou, H.; Wang, C.; Shi, J.; Dong, H. Dislocation Pipe Diffusion of Mn during Annealing of 5 Mn Steel. J. Iron Steel Res. Int. 2016, 23, 1277-1280. [CrossRef]

22. Kirchheim, R. Interaction of hydrogen with dislocations in palladium-I. Activity and diffusivity and their phenomenological interpretation. Acta Metall. 1981, 29, 835-843. [CrossRef]

23. Edalati, K.; Akiba, E.; Horita, Z. High-pressure torsion for new hydrogen storage materials. Sci. Technol. Adv. Mater. 2018, 19, 185-193. [CrossRef] [PubMed]

24. Kittler, M.; Reiche, M. Dislocations as active components in novel silicon devices. Adv. Eng. Mater. 2009, 11, 249-258. [CrossRef]

25. Reiche, M.; Kittler, M.; Uebensee, H.; Pippel, E.; Haehnel, A.; Birner, S. Electronic properties of dislocations. Appl. Phys. A Mater. Sci. Process. 2016, 122, 389. [CrossRef]

26. Ishida, T.; Kakushima, K.; Mizoguchi, T.; Fujita, H. Role of dislocation movement in the electrical conductance of nanocontacts. Sci. Rep. 2012, 2, 21-24. [CrossRef]

27. Sunde, J.K.; Marioara, C.D.; Wenner, S.; Holmestad, R. On the microstructural origins of improvements in conductivity by heavy deformation and ageing of Al-Mg-Si alloy 6101. Mater. Charact. 2021, 176, 111073. [CrossRef]

28. Mendez, J.P.; Ponga, M. MXE: A package for simulating long-term diffusive mass transport phenomena in nanoscale systems. Comput. Phys. Commun. 2021, 260, 107315. [CrossRef]

29. Mishin, Y. Machine-learning interatomic potentials for materials science. Acta Mater. 2021, 214, 116980. [CrossRef]

30. Piggott, S. Colin Renfrew: Before civilization: The radiocarbon revolution and prehistoric Europe. London: Jonathan Cape, 1973. 292 pp., 12 pls., 58 figs. £3.95. Antiquity 1974, 48, 68-69. [CrossRef] 